\title{
Metabolic Syndrome Is Associated with Health-Related Quality of Life in Suspected Patients with Nonalcoholic Steatohepatitis
}

\author{
Ali Gholami ${ }^{a-c}$ Farhad Zamani ${ }^{d}$ Bayan Hosseini ${ }^{e}$ Rahim Sharafkhani ${ }^{f}$ \\ Mansooreh Maadid Zahra Moosavi Jahromig, h ${ }^{\text {h }}$ Maryam Khazaee-Pool ${ }^{i}$ \\ Masoudreza Sohrabi ${ }^{d}$ \\ ${ }^{a}$ Department of Public Health, School of Public Health, Neyshabur University of Medical Sciences, Neyshabur, Iran; \\ ${ }^{b}$ Department of Epidemiology, School of Public Health, Iran University of Medical Sciences, Tehran, Iran; ' ${ }^{C}$ Non-Communicable \\ Diseases Research Center, Endocrinology and Metabolism Research Institute, Tehran University of Medical Sciences, Tehran, \\ Iran; ${ }^{\mathrm{d}}$ Gastrointestinal and Liver Disease Research Center, Firoozgar Hospital, Iran University of Medical Sciences, Tehran, \\ Iran; ${ }^{e}$ Department of Epidemiology, School of Public Health, Tehran University of Medical Sciences, Tehran, Iran; ${ }^{f} \mathrm{PhD}$ in \\ Epidemiology, Khoy University of Medical Sciences, Khoy, Iran; ${ }^{9}$ Health Management and Economics Research Center, Iran

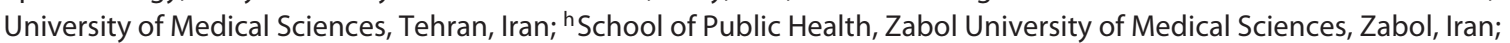 \\ 'Department of Health Education and Promotion, School of Public Health, Zanjan University of Medical Sciences, Zanjan, Iran
}

\section{Significance of the Study}

- In this study, the effect of metabolic syndrome on health-related quality of life (HRQOL) was examined in patients with suspected nonalcoholic steatohepatitis. A significant association between metabolic syndrome and HRQOL was observed in these patients. This finding could be useful in therapy and in devising health policy.

\section{Keywords}

Metabolic syndrome · Nonalcoholic steatohepatitis .

Quality of life · Twelve-Item Short-Form Health Survey

\begin{abstract}
Objective: This study was designed to examine the effect of metabolic syndrome (MetS) on health-related quality of life (HRQOL) in patients with suspected nonalcoholic steatohepatitis (NASH). Subjects and Methods: Three hundred thirtytwo patients (236 males and 96 females) with suspected NASH from the Amol cohort study were included in this study. MetS was diagnosed based on Adult Treatment Panel III criteria and HRQOL was measured using the 12-Item Short-Form
\end{abstract}

\begin{tabular}{ll}
\hline KARGER & ( 2018 The Author(s) \\
& Published by S. Karger AG, Basel \\
E-Mail karger@karger.com & $\begin{array}{l}\text { This is an Open Access article licensed under the Creative Commons } \\
\text { Attribution-NonCommercial-4.0 International License (CC BY-NC) } \\
\text { (http://www.karger.com/Services/OpenAccessicense), applicable to } \\
\text { thww.karger.com/mpp } \\
\text { mercial purposes requires written permission. }\end{array}$
\end{tabular}

Health Survey (SF-12) questionnaire (with 8 subscales and 2 summary components). A multivariable linear regression model was used to assess the independent effect of MetS on HRQOL. Results: The mean age of the study population was $42 \pm 13$ years (range 18-82). The prevalence of MetS was $43.4 \%(n=144)$ and the mean scores on the Physical Component Summary (PCS) and the Mental Component Summary were $72.4 \pm 20.86$ and $42.7 \pm 12.42$, respectively. The multivariable linear regression model showed that MetS was negatively associated with 4 subscales of HRQOL that included: role limitations due to physical problems (RP) $(B=-14.05, p=$ $0.004)$, bodily pain (BP) $(B=-7.37, p=0.02)$, vitality (VT) $(B=$ $-7.72, p=0.022)$, and role limitations due to emotional problems (RE) ( $B=-12.67, p=0.005)$ after adjustment for other
Masoudreza Sohrabi

Beh Afarin Ave., Valiasr Sq. Tehran (Iran)

E-Mail Sohrab_r@yahoo.com 
variables. Also, MetS had a borderline association with the general health and mental health subscales and the PCS ( $p<$ 0.1 ). Conclusion: In this study, there was a strong association between MetS and 4 subscales (RP, BP, VT, and RE) of HRQOL in patients with suspected NASH; this could be considered as a part of health policy to improve general health.

(c) 2018 The Author(s)

Published by S. Karger AG, Basel

\section{Introduction}

With the increasing trend toward overweight and obesity in developing countries [1], health policy-makers are progressively faced with the problems and dilemmas related to nonalcoholic fatty liver disease (NAFLD) [2]. The spectrum of this disease ranges from simple steatosis to cirrhosis and eventually hepatocellular carcinoma. Currently NAFLD is considered the most prevalent chronic liver disease in many countries including Iran [3]. NAFLD is a complex metabolic condition in which both lifestyle and genetic factors have a pathogenic role and it has been increasingly recognized as a major cause of liver-related morbidity and mortality $[4,5]$. In this setting, nonalcoholic steatohepatitis (NASH) is histologically determined by hepatic steatosis and cellular damage with features of inflammation and/or fibrosis $[6,7]$. NASH may progress to liver fibrosis or cirrhosis and it is likely that this disease could become the most common cause of liver-related mortality in the near future [8]. The pathogenesis of NAFLD/NASH is not clear, and many hypotheses had been proposed. Obesity, diabetes mellitus, and dyslipidemia have been reported as predisposing risk factors for NASH [7]. Due to the variety in the pathogenesis of NAFLD, its clinical presentation may be different. Many patients with NAFLD/NASH are asymptomatic [9]. Some comorbidities such as metabolic syndrome (MetS) and obesity might occur in NAFLD/NASH patients [10], but MetS is a common comorbidity in subjects with NAFLD/ NASH and could affect their health-related quality of life (HRQOL). There have been only a few studies (especially in our region) assessing the link between MetS and HRQOL in NAFLD/NASH patients [11, 12]. Most studies have evaluated MetS status and QOL in NAFLD/ NASH patients separately. Also, considering the social and economic burden of NAFLD/NASH, it is estimated that knowing the HRQOL and associated factors (especially MetS) of patients could be of great importance in health policy-making as well as in therapy.

The hypothesis of this study was that HRQOL scores in suspected NASH patients would be associated with
MetS. Thus, the aim was to evaluate the effects of MetS on HRQOL, and to investigate whether or not the demographics and clinical data including sex, age, marital status, occupational status, exercise status, BMI, history of smoking, history of drug abuse, history of alcohol consumption, history of diabetes, and hypertension would moderate this relationship.

\section{Subjects and Methods}

\section{Design and Sample}

This cross-sectional study was conducted within the framework of Amol cohort health study (phase 1; 2008-2011). In this cohort, a total of 7,104 subjects aged 10-90 years were selected by cluster random sampling from Amol healthcare centers. Nine hundred sixty persons at baseline were excluded due to unwillingness to participate, moving, or pregnancy. Thus, this study was conducted in 6,140 participants. All of them underwent a complete physical examination and fasting blood samples were obtained for assessment of fasting blood sugar and the lipid profile. A transabdominal ultrasonography was performed on each participant. Ultrasonography was performed by a senior radiologist using previously published methods [5]. Subjects with any signs or symptoms of liver diseases were referred for more specific evaluations.

\section{Definition of Suspected NASH}

Liver biopsy remains the gold standard for diagnosis of the degree of hepatosteatosis and inflammatory activity and NASH [13]. Also a previous study revealed that age, glucose level, diastolic blood pressure, systolic blood pressure, BMI, and lipid profile are associated with the diagnosis of NAFLD [14]. Because liver biopsy is an invasive technique it was not done in the present study, but patients with fatty liver on transabdominal ultrasonography along with an elevated serum level of alanine aminotransferase without any other liver diseases were considered "suspected" NASH patients.

\section{Definition of MetS}

MetS was defined based on fulfillment of at least 3 of the 5 components of the National Cholesterol Education Program Adult Treatment Panel III criteria as follows [15]: waist circumference > $102 \mathrm{~cm}$ (40 in) for males and $>88 \mathrm{~cm}$ (35 in) for females, blood pressure $\geq 130 / \geq 85 \mathrm{~mm} \mathrm{Hg}$, fasting glucose $\geq 100 \mathrm{mg} / \mathrm{dL}$, triglycerides $\geq 150 \mathrm{mg} / \mathrm{dL}$, and HDL cholesterol $<40 \mathrm{mg} / \mathrm{dL}$ for males and $<50 \mathrm{mg} / \mathrm{dL}$ for females.

\section{Study Population}

Patients were selected based on our definition of suspected NASH. Exclusion criteria were: (1) age $<18$ years, (2) having other known liver diseases, and (3) unwillingness to participate in this study. The research was explained to all of the participants and written consent was obtained from each one.

Three hundred thirty-nine subjects with suspected NASH were detected from all of the participants (6,140 persons). Seven questionnaires had more than $20 \%$ missing data and thus were excluded from this study. Therefore, 332 subjects with suspected NASH who had complete sociodemographic, clinical, and HRQOL data were recruited for data analysis. 
Table 1. Characteristics of the study population

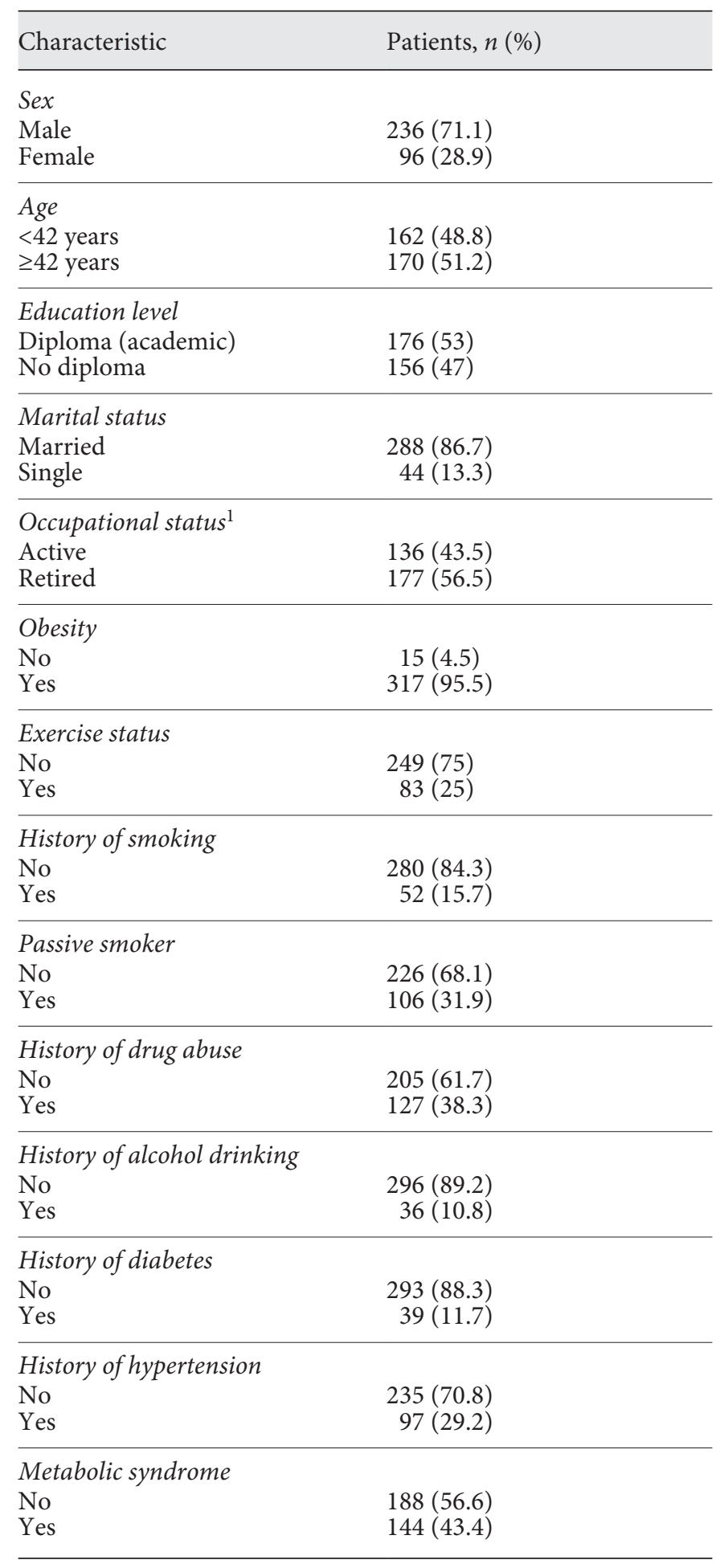

There were 332 patients in total. ${ }^{1}$ Some missing data exist for this variable.
Data Collection

Twelve-Item Short-Form Questionnaire

The 12-Item Short-Form Questionnaire (SF-12) is a multidimensional questionnaire on HRQOL. This questionnaire had been previously developed and tested among the general Iranian population [16]. The SF-12 contains a Likert scale format including 12 items and $8 \mathrm{HRQOL}$ subscales derived from aggregated items. The SF-12 subscales were: (1) physical functioning (2 items), (2) role limitations due to physical problems (RP) (2 items), (3) bodily pain (BP) (1 item), (4) general health (1 item), (5) vitality (VT) (1 item), (6) social functioning (SF) (1 item), (7) role limitations due to emotional problems (RE) (2 items), and (8) mental health (MH) (2 items). In summary, subscales $1-4$ covered the Physical Component Summary (PCS) and subscales 5-8 covered the Mental Component Summary (MCS). The items were computed based on scores of $0-100$, where 0 indicated the lowest level of health measured and 100 indicated the highest level of health measured [17]. Eight subscales and 2 summary components of the SF-12 questionnaire were considered as dependent variables.

Sociodemographic and Clinical Factors Questionnaire (except MetS and Its Components)

Demographic and clinical data included gender (male or female), age ( $\leq 42$ years or $>42$ years), marital status (married or single), occupational status (retired or active), exercise status (having daily regular physical activity; yes or no), obesity (yes or no), history of smoking (yes or no), history of drug abuse (yes or no), history of alcohol consumption (yes or no), history of diabetes (yes or no), and history of hypertension (yes or no) and were considered covariates in this study.

\section{Statistical Analysis}

Statistical evaluations were performed using IBM SPSS Statistics for Windows (SPSS), version 20.0 (IBM Corp., Armonk, NY, USA). A descriptive analysis (frequencies, percentages, ranges, means, and $\mathrm{SD})$ of the sociodemographic and clinical factors is reported. The Kolmogorov-Smirnov test was used to assess the normality of the data. The Pearson correlation coefficient was used to determine the level of agreement between the 8 subscales of the SF-12. The univariate test ( $t$ independent) was used to investigate the association between subjects' HRQOL and independent and covariate variables in the first step. Multivariable linear regression models (with the backward method) were used to assess the independent effect of MetS on HRQOL. Transformed scores were used for statistical analyses in all subscales. $p<0.2$ in univariate analyses and $p<0.05$ in multivariable analyses were considered statistically significant.

\section{Results}

Demographic and other pertinent data are shown in Table 1 . The mean age of the study population was $42 \pm$ 13 years (range $18-82$ ), the mean BMI was $31.5 \pm 4.7$, and 144 (43.4\%) subjects had MetS.

Among the different subscales of the SF-12, the lowest and highest mean scores were observed in SF subscale (mean: $20.9 \pm 25.6$ ) and the BP subscale (mean: 77.8 \pm 27.9 ), 
Table 2. Correlation coefficients for the PCS, the MCS and the 8 subscales of the SF-12

\begin{tabular}{|c|c|c|c|c|c|c|c|c|c|c|}
\hline & $\mathrm{PF}$ & $\mathrm{RP}$ & BP & $\mathrm{GH}$ & VT & SF & $\mathrm{RE}$ & $\mathrm{MH}$ & MCS & PCS \\
\hline PF & 1 & $0.661^{* *}$ & $0.553^{* *}$ & $-0.287^{* *}$ & $-0.429^{* *}$ & $-0.348^{* *}$ & $0.465^{* *}$ & $-0.410^{* *}$ & $-0.229^{* *}$ & $0.833^{* *}$ \\
\hline $\mathrm{RP}$ & & 1 & $0.438^{* *}$ & $-0.226^{* *}$ & $-0.391^{* *}$ & $-0.324^{* *}$ & $0.534^{* *}$ & $-0.425^{* *}$ & $-0.152^{*}$ & $0.822^{* *}$ \\
\hline BP & & & 1 & $-0.521^{* *}$ & $-0.397^{* *}$ & $-0.410^{* *}$ & $0.350^{* *}$ & $-0.440^{* *}$ & $-0.345^{* *}$ & $0.605^{* *}$ \\
\hline $\mathrm{GH}$ & & & & 1 & $0.341^{* *}$ & $0.135^{*}$ & $-0.169^{*}$ & $0.291^{* *}$ & $0.262^{* *}$ & -0.025 \\
\hline VT & & & & & 1 & $0.358^{* *}$ & $-0.390^{* *}$ & $0.556^{* *}$ & $0.674^{* *}$ & $-0.371^{* *}$ \\
\hline $\mathrm{MH}$ & & & & & & & & 1 & $0.538^{* *}$ & $-0.429^{* *}$ \\
\hline MCS & & & & & & & & & 1 & $-0.198^{* *}$ \\
\hline PCS & & & & & & & & & & 1 \\
\hline
\end{tabular}

PF, physical functioning; RP, role limitations due to physical problems; BP, bodily pain; GF, general health; VT, vitality; SF, social functioning; RE, role limitations due to emotional problems; $\mathrm{MH}$, mental health; PCS, Physical Component Summery; MCS, Mental Component Summary; SF-12, 12-Item Short-Form Health Survey. ${ }^{*}$ Correlation is significant at the $<0.05$ level (2-tailed). ${ }^{* *}$ Correlation is significant at the $<0.001$ level (2-tailed).

Fig. 1. Subscales and components of healthrelated quality of life (HRQOL) in the study population. PF, physical functioning; RP, role limitations due to physical problems; $\mathrm{BP}$, bodily pain; $\mathrm{GH}$, general health; VT, vitality; SF, social functioning; RE, role limitations due to emotional problems; $\mathrm{MH}$, mental health; PCS, Physical Component Summary; MCS, Mental Component Summary.

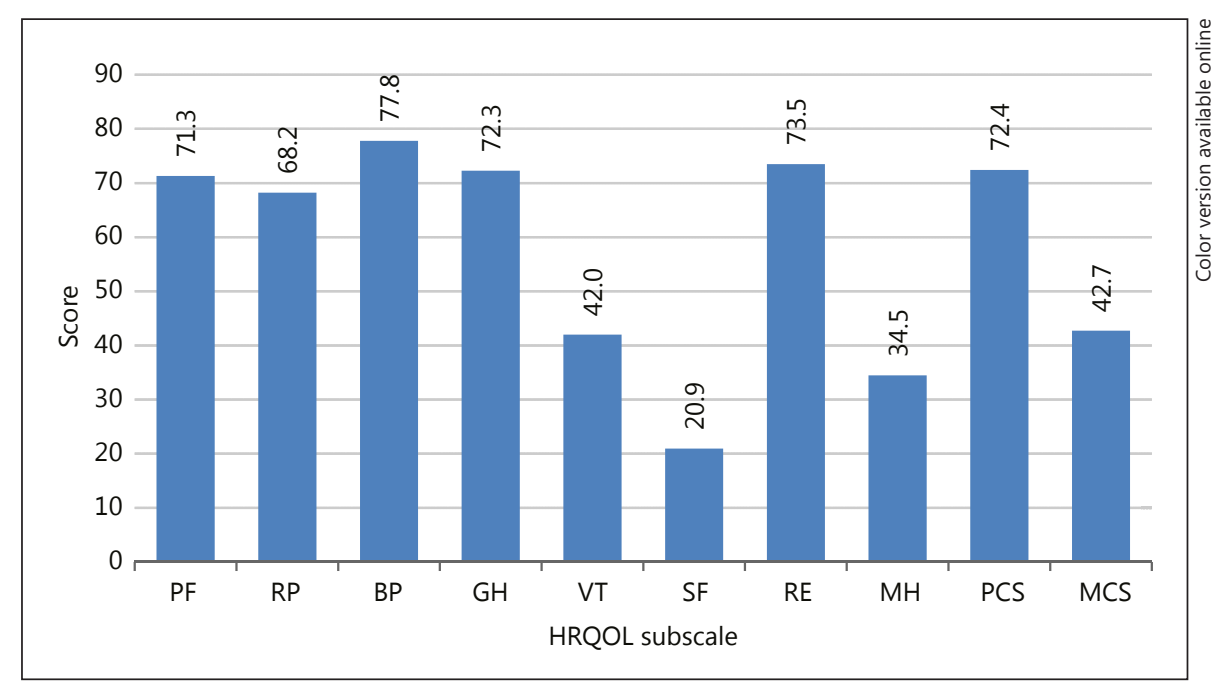

respectively. Also, the mean scores on the PCS and MCS were $72.4 \pm 20.86$ and $42.7 \pm 12.42$, respectively (Fig. 1).

The correlations between the 8 subscales of the SF- 12 are presented in Table 2; as can be observed, there were statistically significant correlations between all of the subscales. There were also statistically significant correlations between the summary components (PCS and MCS) and the scores obtained from the majority of the subscales.

For sociodemographic and clinical factors, a using $t$ independent test, age, marital status, occupational status, exercise status, BMI, history of drug abuse, history of diabetes, and history of hypertension (Table 3 ) were related to some subscales of HRQOL $(p<0.2)$. MetS was related to more subscales of HRQOL. Gender and passive smok- er were not related to any subscales in the univariate analysis $(p \geq 0.2)$.

In the multivariable analysis, MetS was significantly related to four subscales of HRQOL including: $\mathrm{RP}(\mathrm{B}=$ $-14.05, p=0.004), \mathrm{BP}(\mathrm{B}=-7.37, p=0.02), \mathrm{VT}(\mathrm{B}=-7.72$, $p=0.022)$ and RE (B: $-12.67, p=0.005)$ after adjustment for other variables. Borderline relationships were observed in the general health and $\mathrm{MH}$ subscales and the PCS. A borderline relationship was also observed between exercise activity and the MH subscale $(B=-4.70$, $p=0.087)$, between occupational status and the RP subscale $(B=-8.38, p=0.083)$, and between exercise status and the physical functioning subscale $(B=7.73, p=$ 0.099). A borderline relationship was observed between 
Table 3. Mean scores of the different subscales of HRQOL in the study population

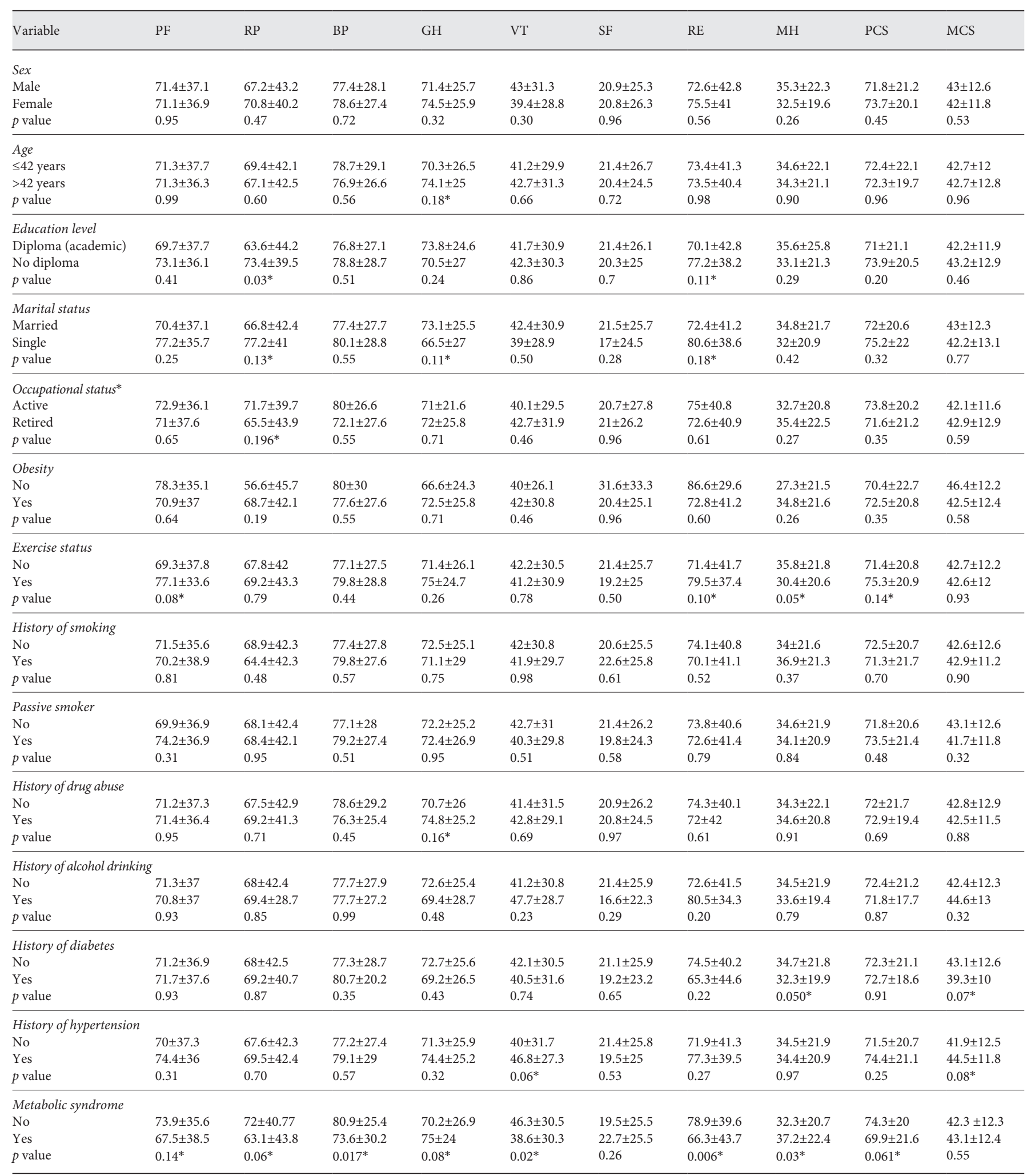

HRQOL, health-related quality of life; PF, physical functioning; RP, role limitations due to physical problems; BP, bodily pain; GF, general health; VT, vitality; SF, social functioning; RE, role limitations due to emotional problems; MH, mental health; PCS, Physical Component Summary; MCS, Mental Component Summary. ${ }^{*}$ Significant at $p<0.2$. 
Table 4. Multivariable analysis of independent variables in relation to 8 dimensions and 2 summary components of HRQOL

\begin{tabular}{|c|c|c|c|c|c|c|c|c|c|c|}
\hline Independent variables & \multicolumn{10}{|c|}{ QOL subscale } \\
\hline Age (>42 years) & - & - & - & 0.68 & - & - & - & - & - & - \\
\hline Educational level (no diploma) & - & 0.24 & - & - & - & - & 0.44 & - & - & - \\
\hline Marital status (single) & - & 0.17 & - & 0.12 & - & - & 0.25 & - & - & - \\
\hline Exercise status (yes) & $0.099^{*}$ & - & - & - & - & - & 0.22 & $0.076^{*}$ & 0.22 & - \\
\hline History of smoking (yes) & - & - & - & - & - & - & - & - & - & - \\
\hline Passive smoker (yes) & - & - & - & - & - & - & - & - & - & - \\
\hline History of drug abuse (yes) & - & - & - & 0.33 & - & - & - & - & - & - \\
\hline History of alcohol drinking (yes) & - & - & - & - & - & - & - & - & - & - \\
\hline
\end{tabular}

HRQOL, health-related quality of life; PF, physical functioning; RP, role limitations due to physical problems; BP, bodily pain; GF, general health; VT, vitality; SF, social functioning; RE, role limitations due to emotional problems; MH, mental health; PCS, Physical Component Summery; MCS, Mental Component Summery. * Significant at $p<0.1$. ** Significant at $p<0.05$.

diabetes $(B=-3.86, p=0.067)$ and hypertension $(B=2.59$, $p=0.083)$ in relation to the MCS. Age, education level, marital status, obesity, and drug use did not have any relationship with the HRQOL subscales or the summary components $(p>0.05)$ (Table 4$)$.

\section{Discussion}

In the present study, the mean BP and SF scores were highest and lowest among the SF-12 subscales. Thus, in approximately $23 \%$ of the participants, the mean BP subscale score implied that pain interfered with their normal work and the mean SF subscale score implied that approximately $79 \%$ of the participants had a problem engaging in social interactions, interpersonal relationships, and activities of independent living. Moreover, the mean MCS score was lower than the mean PCS score. Therefore, we can conclude that mental health problems were more important than physical problems. Furthermore, our finding regarding MCS and PCS did not concur with studies by Sayiner et al. [18], David et al. [19], and Afendy et al. [20]. Our study indicates impairment of HRQOL among patients with suspected NASH that is comparable with previous reports $[21,22]$. In their studies, Golabi et al. [23] and Sarrafzadegan et al. [24] illustrated a noticeable QOL impairment in NAFLD [23, 24].

MetS and Quality of Life in Suspected NASH Patients
MetS is a chronic and progressive condition that influences different aspects of the personal health status such as physical, mental, and even sexual functions $[25,26]$. The prevalence of MetS in countries such as Iran has been increasing in recent years [27]. As a result, it may have an impact on mental and physical statuses and, eventually, QOL. Despite the many studies on MetS, studies regarding MetS and QOL are limited, particularly among patients with NAFLD/NASH. In the present study, we showed that MetS is significantly associated with decreased RP, BP, VT, and RE subscale scores after adjusting for other study variables. It also had a borderline association with general health, MH, and the PCS. Sarrafzadegan et al. [24], in a population-based study, observed a significant difference between the physical health domain of HRQOL in women with and those without MetS [24].

The effect of MetS on the health consequences of patients with NAFLD/NASH is an understudied determinant of health situations, but enhancing the awareness of the medical community about the harmful prevalence of MetS among patients with NAFLD/NASH is essential. More detailed studies are needed to promote our knowledge on the relationship between MetS and the HRQOL of patients with NAFLD/NASH to develop programs for improving HRQOL among these patients. In addition, previous studies have revealed that visceral fat as a risk factor for developing insulin resistance has a great influ- 
ence on the development of Mets and NAFLD. Therefore, as a result, the reduction of visceral fat may influence the improvement of MetS and its adverse effects, such as NAFLD and cardiovascular disease [28, 29].

The limitations of this study include its cross-sectional nature and not using factors, such as local residence and ethnicity, that might have impacted the results. The SF-12 is a self-reported HRQOL instrument that might be affected by factors that are not related to MetS.

\section{Conclusion}

In this study, patients with suspected NASH showed a relatively strong association between MetS and the scores on 4 subscales (RP, BP, VT, and RE) of HRQOL, which could consequently play a role in advancing the disease. Therefore, HRQOL might play an important role in the development of NAFLD/NASH.

\section{Acknowledgments}

The authors would like to thank the staff of Amol Hefdah Shahrivar Hospital and members of the Amol healthcare centers and their administrators for participating in this project. We thank Reza Pirzad (radiologist) and Fateme Sima Saeedian (endocrinologist) for their technical assistance.

\section{Disclosure Statement}

None.

\section{References}

1 Arroyo-Johnson C, Mincey KD: Obesity epidemiology worldwide. Gastroenterol Clin North Am 2016;45:571-579.

2 Huh JH, Kim KJ, Kim SU, et al: Obesity is more closely related with hepatic steatosis and fibrosis measured by transient elastography than metabolic health status. Metabolism 2017;66:23-31.

3 Rowe IA: Lessons from epidemiology: the burden of liver disease. Dig Dis 2017;35:304309.

4 Sohrabpour A, Rezvan H, Amini-Kafiabad S, et al: Prevalence of nonalcoholic steatohepatitis in Iran: a population based study. Middle East J Dig Dis 2010;2:14-19.

5 Hadizadeh F, Faghihimani E, Adibi P: Nonalcoholic fatty liver disease: diagnostic biomarkers. World J Gastrointest Pathophysiol 2017;8:11-26.

6 Doulberis M, Kotronis G, Gialamprinou D, et al: Non-alcoholic fatty liver disease: an update with special focus on the role of gut microbiota. Metabol 2017;71:182-197.

7 Mazzotti A, Caletti MT, Sasdelli AS, et al: Pathophysiology of nonalcoholic fatty liver disease: lifestyle-gut-gene interaction. Dig Dis 2016;34(suppl 1):3-10.

8 Goh GB, Pagadala MR, Dasarathy J, et al: Clinical spectrum of non-alcoholic fatty liver disease in diabetic and non-diabetic patients. BBA Clin 2015;3:141-145.

9 Khoonsari M, Mohammad Hosseini Azar M, Ghavam R, et al: Clinical manifestations and diagnosis of non-alcoholic fatty liver disease. Iran J Pathol 2017;12:99-105.

10 Tarantino G, Finelli C: What about non-alcoholic fatty liver disease as a new criterion to define metabolic syndrome? World J Gastroenterol 2013;19:3375-3384.
11 Dan AA, Kallman JB, Wheeler A, et al: Healthrelated quality of life in patients with non-alcoholic fatty liver disease. Aliment Pharmacol Ther 2007;26:815-820.

12 Mishra A, Younossi ZM: Epidemiology and natural history of non-alcoholic fatty liver disease. J Clin Exp Hepatol 2012;2:135-144.

13 Spengler EK, Loomba R: Recommendations for diagnosis, referral for liver Biopsy, and treatment of NAFLD and NASH. Mayo Clin Proc 2015;90:1233-1246.

14 Li S, Su L, Lv G, et al: Transabdominal ultrasonography of the pancreas is superior to that of the liver for detection of ectopic fat deposits resulting from metabolic syndrome. Medicine (Baltimore) 2017;96:e8060.

15 Grundy SM, Hansen B, Smith SC Jr, et al: Clinical management of metabolic syndrome. Circulation 2004;109:551-556.

16 Montazeri A, Vahdaninia M, Mousavi SJ, et al: The Iranian version of 12 -Item Short Form Health Survey (SF-12): factor structure, internal consistency and construct validity. BMC Public Health 2009;9:341.

17 Gandhi SK, Salmon JW, Zhao SZ, et al: Psychometric evaluation of the 12-Item ShortForm Health Survey (SF-12) in osteoarthritis and rheumatoid arthritis clinical trials. Clin Ther 2001;23:1080-1098.

18 Sayiner M, Stepanova M, Pham H, et al: Assessment of health utilities and quality of life in patients with non-alcoholic fatty liver disease. BMJ Open Gastroenterol 2016;3:e000106.

19 David K, Kowdley KV, Unalp A, et al: Quality of life in adults with nonalcoholic fatty liver disease: baseline data from the nonalcoholic steatohepatitis clinical research network. Hepatology 2009;49:1904-1912.
20 Afendy A, Kallman JB, Stepanova M, et al: Predictors of health-related quality of life in patients with chronic liver disease. Aliment Pharmacol Ther 2009;30:469-476.

21 Younossi ZM, Zheng L, Stepanova M, et al: Trends in outpatient resource utilizations and outcomes for Medicare beneficiaries with nonalcoholic fatty liver disease. J Clin Gastroenterol 2015;49:222-227.

22 Younossi ZM, Boparai N, Price LL, et al: Health-related quality of life in chronic liver disease: the impact of type and severity of disease. Am J Gastroenterol 2001;96:2199-2205.

23 Golabi P, Otgonsuren M, Cable R, et al: Nonalcoholic fatty liver disease (NAFLD) is associated with impairment of health related quality of life (HRQOL). Health Qual Life Outcomes 2016;14:18.

24 Sarrafzadegan N, Gharipour M, Ramezani MA, et al: Metabolic syndrome and healthrelated quality of life in Iranian population. J Res Med Sci 2011;16:254-261.

25 Gardner AW, Montgomery PS: The effect of metabolic syndrome components on exercise performance in patients with intermittent claudication. J Vasc Surg 2008;47:1251-1258.

26 Esposito K, Giugliano F, Ciotola M, et al: Obesity and sexual dysfunction, male and female. Int J Impot Res 2008;20:358-365.

27 Dalvand S, Niksima SH, Meshkani R, et al: Prevalence of metabolic syndrome among Iranian population: a systematic review and metaanalysis. Iran J Public Health 2017;46:456-467.

28 Finelli C, Tarantino G: Should visceral fat, strictly linked to hepatic steatosis, be depleted to improve survival? Hepatol Int 2013;7:413-428.

29 Finelli C, Sommella L, Gioia S, et al: Should visceral fat be reduced to increase longevity? Ageing Res Rev 2013;12:996-1004. 